\title{
GENERALIZED BEATTY SEQUENCES
}

\author{
A. MCD. MERCER \\ Department of Mathematics and Statistics \\ University of Guelph \\ Guelph, Ontario, Canada NIG 2WI
}

(Recieved April 12, 1978)

ABSTRACT. A well-known result due to $S$. Beatty is that if $\alpha$ and $\beta$ are positive irrational numbers satisfying $\alpha^{-1}+\beta^{-1}=1$ then each positive integer is to be found in precisely one of the sequences $\{[k \alpha]\},\{[k \beta]\}(k=1,2,3, \ldots)$ where $[x]$ denotes the integral part of $\mathrm{x}$. The present note generalizes this result to the case of the pair of sequences $\{[f(k)]\},\{[g(k)]\}$ with suitable hypotheses on the functions $f$ and $g$. The special case $f(x)=\alpha x, g(x)=\beta x$ is the result due to Beatty.

KEY WORDS AND PHRASES. Partitions, Complementary systems of integers, integer sequences.

AMS (MOS) SUBJECT CLASSIFICATION (1970) CODES. Primary 10A45, Secondary $10 L 99$.

1. The object of this note is to prove the following result.

THEOREM. Let $f$ and $g$ be strictly increasing in $[0,+\infty)$ with $f(0)=g(0)=0$. Let $\mathrm{f}^{\prime}$ exist in $(0,+\infty)$ and suppose $1<\mathrm{f}^{\prime}(\mathrm{x})<2$ there. Suppose also that 
$f(k), g(k)(k=1,2,3, \ldots)$ are never integral. Writing $\phi \equiv f^{-1}$ and $\theta \equiv g^{-1}$ let $\phi(x)+\theta(x)=x$ in $[0,+\infty)$. Then each positive integer is to be found in precisely one of the sequences $\{[f(k)]\},\{[g(k)]\}(k=1,2,3, \ldots)$ and these sequences consist of distinct integers.

2. This theorem generalizes a well-known result. Namely, if $\alpha$ and $\beta$ are positive irrationals satisfying $\alpha^{-1}+\beta^{-1}=1$ then each positive integer is to be found in precisely one of the sequences $\{[k \alpha]\},\{[k \beta]\}(k=1,2,3, \ldots)$.

This latter result appears to be due originally to Beatty [1] and has lead to notes by several authors, one of the latest being Fraenke1 [2] who also gives a fairly complete bibliography. Two additional references are given at the end of the present note.

In [2] Fraenkel gives also a simple proof of a result due to Lambek and Moser. This result characterizes "two-way splittings" of the set of integers in the sense that it indicates how the second sequence can be generated when an origional sequence of integers is given. Their result is of quite a different nature from the one we prove here. Our present theorem seems to represent a generalization previously overlooked.

3. In this section we prove the theorem stated in Section 1. The proof falls into three parts.

PROOF. (a) The two sequences of the theorem are disjoint. Suppose on the contrary that there are integers $m$ and $n$ such that $[f(m)]=$ $[g(n)]$. Then for some $\varepsilon, \delta \in(0,1)$ and some integer $M$ we will have $f(m)=M+\varepsilon$, $g(n)=M+\delta$.

Hence $\quad m+n=\phi(M+\varepsilon)+\theta(M+\delta)$.

Let us assume, without loss of generality, that $\varepsilon \leqq \delta$. Since $\phi$ and $\theta$ are strictly increasing functions we get

$$
\phi(M+\varepsilon)+\theta(M+\varepsilon) \leqq m+n \leqq \phi(M+\delta)+\theta(M+\delta)
$$


That is $M+\varepsilon \leqq m+n \leqq M+\delta,(0<\varepsilon \leqq \delta<1)$ which is impossible.

(b) No integer is absent from both sequences.

Suppose on the contrary that the integer $\mathrm{N}$ is omitted from both sequences. Let $k$ be any integer and consider $[f(k)]$ and $[f(k+1)]$. If $[f(k)]=Q$ then

$$
f(k)=Q+\varepsilon \quad(0<\varepsilon<1)
$$

$$
\begin{aligned}
& \text { Hence } f(k+1)=f(k)+f(k+1)-f(k) \\
& =Q+\varepsilon+f^{\prime}(k+\gamma) \quad(0<\gamma<1) \\
& =Q+\Delta \quad \text { (Say) }
\end{aligned}
$$

where $1<\Delta<3$, since $1<f^{\prime}(x)<2$ by hypothesis.

Hence $[f(k+1)]=Q+1$ or $Q+2$.

It follows that if an integer $\mathrm{N}$ is omitted from both sequences then it must lie between the integers $[f(n)]$ and $[f(n+1)]$ for some $n$.

Now let $m$ be the greatest integer such that $[g(m)]<[f(n)]$. Since $N-1=[f(n)]$ and $N+1=[f(n+1)]$ we must have $[g(m)] \leqq N-2$ and $[g(m+1)] \geqq N+2$.

That is $\quad \mathrm{f}(\mathrm{n})<\mathrm{N}, \mathrm{g}(\mathrm{m})<\mathrm{N}-1$

and

$$
f(n+1)>N+1, g(m+1)>N+2
$$

From (1) we get

$\mathrm{m}+\mathrm{n}<\phi(\mathrm{N})+\theta(\mathrm{N}-1)$

Therefore

$m+n+\{\theta(N)-\theta(N-1)\}<N$

Since $\theta$ is strictly increasing this gives $m+n+1 \leqq N$

From (2) we get

$\mathrm{m}+\mathrm{n}+2>\phi(\mathrm{N}+1)+\theta(\mathrm{N}+2)$

Therefore

$\mathrm{m}+\mathrm{n}+1>\mathrm{N}+\{\theta(\mathrm{N}+2)-\theta(\mathrm{N}+1)\}$

Since $\theta$ is strictly increasing this gives $m+n \geq N$

The inequalities (3) and (4) provide the desired contradiction and the proof of part (b) is complete.

(c) Each sequence consists of distinct elements.

In the proof of part (b) we have seen that because $1<\mathrm{f}^{\prime}(\mathrm{x})<2$ then $[\mathrm{f}(\mathrm{k})]<[\mathrm{f}(\mathrm{k}+1)]$. Now $1<\mathrm{f}^{\prime}(\mathrm{x})<2$ implies $2<\mathrm{g}^{\prime}(\mathrm{x})<+\infty$ and this, in a 
similar way, implies that $[\mathrm{g}(\mathrm{k})]<[\mathrm{g}(\mathrm{k}+1)]$. This completes the proof of (c) and hence of the theorem.

We conclude this note by mentioning that the special case: $f(x)=\alpha x$, $g(x)=\beta x, \alpha$ irrational, $\alpha^{-1}+\beta^{-1}=1,1<\alpha<2$, is the classical result due to Beatty.

\section{REFERENCES}

1. Beatty, S. Problem 3173, Amer. Math Monthly 33 (1926) 159.

2. Fraenke1, A. S. Complementary Systems of Integers, Amer. Math. Month1y 84 (1977) 114-115.

3. Bang, T. On the sequence $[\mathrm{n} \alpha], \mathrm{n}=1,2, \ldots$, Math Scand. $\underline{5}$ (1957) 69-76.

4. Golomb, S. W. The "Sales Tax" theorem, Math Mag. 49 (1976) 187-189. 


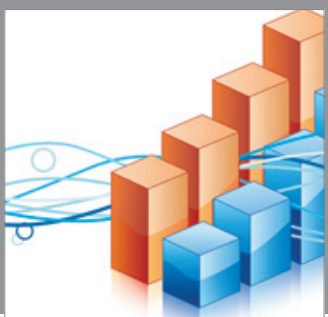

Advances in

Operations Research

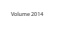

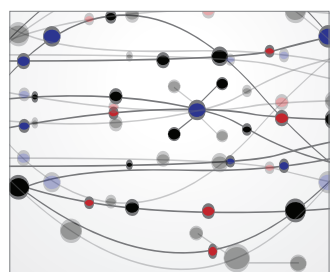

\section{The Scientific} World Journal
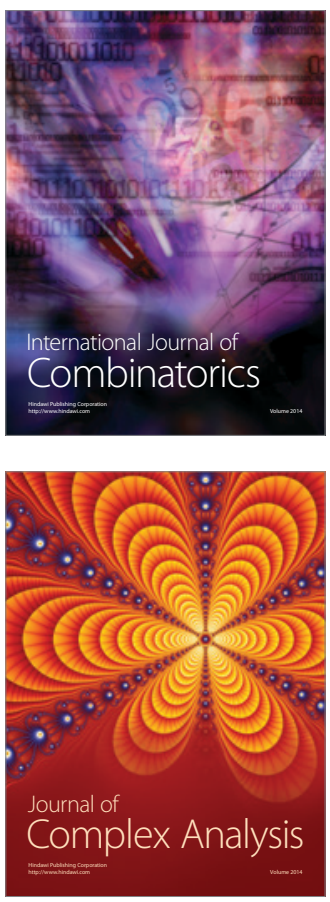

International Journal of

Mathematics and

Mathematical

Sciences
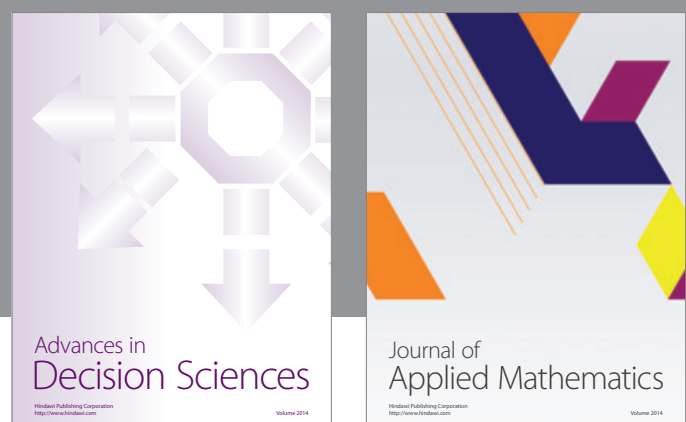

Journal of

Applied Mathematics
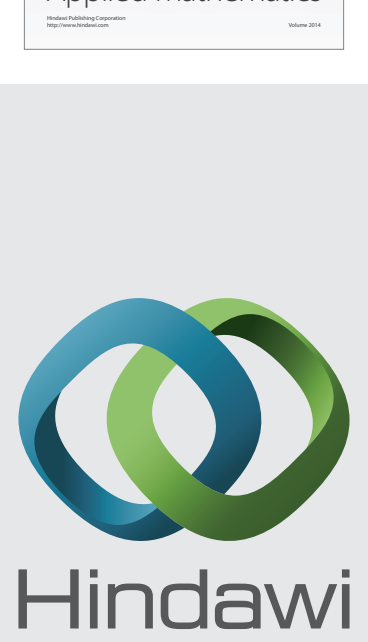

Submit your manuscripts at http://www.hindawi.com
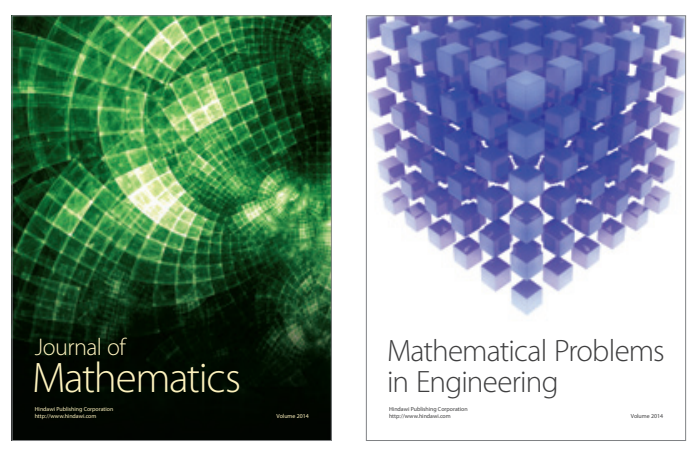

Mathematical Problems in Engineering
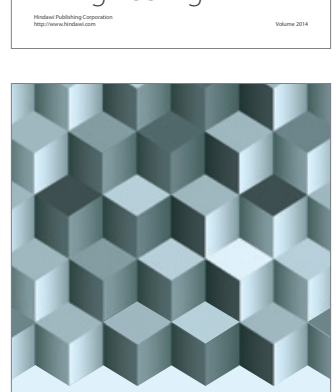

Journal of

Function Spaces
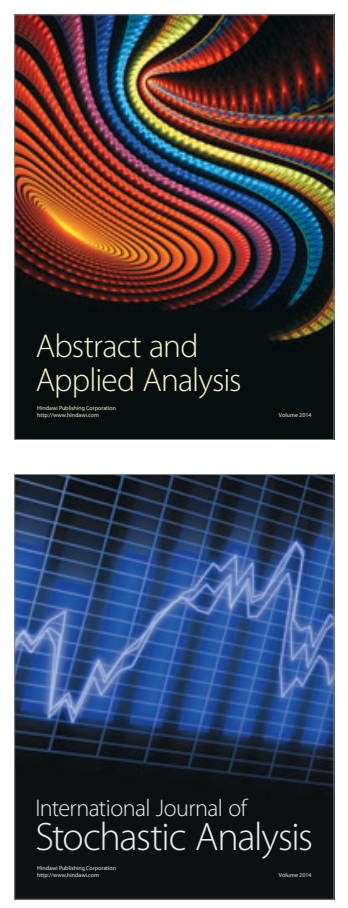

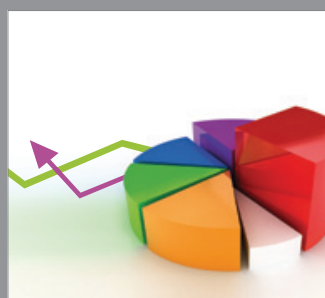

ournal of

Probability and Statistics

Promensencen
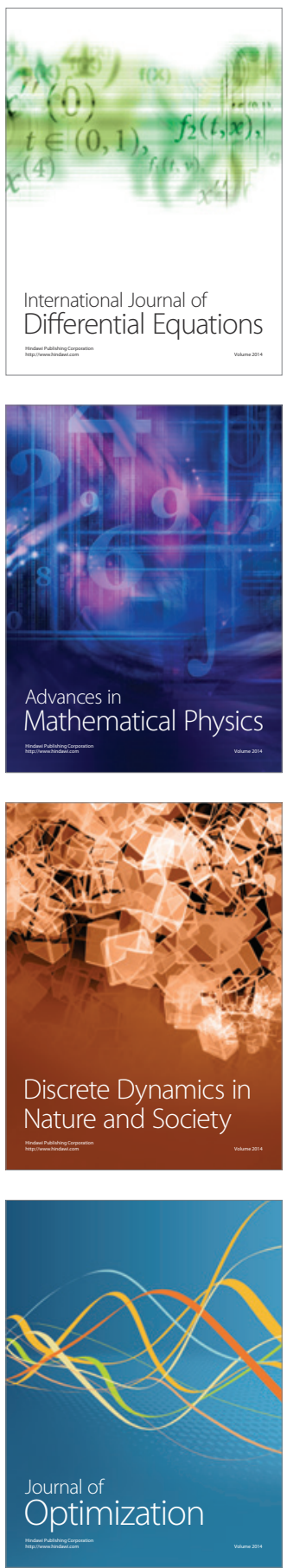